WellBeing International

WBI Studies Repository

6-2013

\title{
Can Acute Dermal Systemic Toxicity Tests Be Replaced With Oral Tests? A Comparison of Route-Specific Systemic Toxicity and Hazard Classifications Under the Globally Harmonized System of Classification and Labelling of Chemicals (GHS)
}

\author{
Nigel P. Moore \\ Dow Europe $\mathrm{GmbH}$ \\ David J. Andrew \\ TSGE LLP \\ Donald L. Bjerke \\ Proctor \& Gamble \\ Stuart Creton \\ National Centre for the Replacement, Refinement and Reduction of Animals in Research \\ David Dreher \\ Covance Laboratories
}

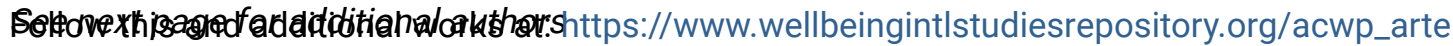

Part of the Bioethics and Medical Ethics Commons, Laboratory and Basic Science Research Commons, and the Research Methods in Life Sciences Commons
\end{abstract}

\section{Recommended Citation}

Moore, N. P., Andrew, D. J., Bjerke, D. L., Creton, S., Dreher, D., Holmes, T., ... \& Rowan, T. G. (2013). Can acute dermal systemic toxicity tests be replaced with oral tests? A comparison of route-specific systemic toxicity and hazard classifications under the Globally Harmonized System of Classification and Labelling of Chemicals (GHS). Regulatory Toxicology and Pharmacology, 66(1), 30-37.

This material is brought to you for free and open access by WellBeing International. It has been accepted for inclusion by an authorized administrator of the WBI Studies Repository. For more information, please contact wbisr-info@wellbeingintl.org.

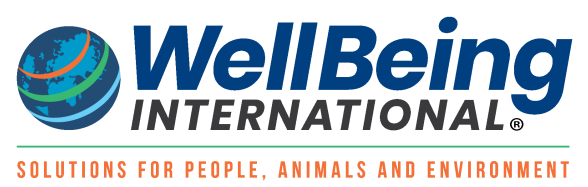




\section{Authors}

Nigel P. Moore, David J. Andrew, Donald L. Bjerke, Stuart Creton, David Dreher, Thomas Holmes, Pilar Prieto, Troy Seidle, and Tim G. Rowan 
Can acute dermal systemic toxicity tests be replaced with oral tests? A comparison of route-specific systemic toxicity and hazard classifications under the Globally Harmonized System of Classification and Labelling of Chemicals (GHS)

Nigel P. Moore ${ }^{a}$, David J. Andrew ${ }^{b}$, Donald L. Bjerke ${ }^{c}$, Stuart Creton ${ }^{d, 1}$, David Dreher ${ }^{\mathrm{e}}$, Thomas Holmes $^{\dagger}$, Pilar Prieto ${ }^{g}$, Troy Seidle ${ }^{\mathrm{h}}$, Tim G. Rowan ${ }^{\mathrm{i}, *}$

${ }^{a}$ Dow Europe $\mathrm{GmbH}$, Horgen, Switzerland

${ }^{\mathrm{b}}$ TSGE LLP, Knaresborough, UK

${ }^{\mathrm{c}}$ Procter and Gamble, Cincinnati, OH, USA

${ }^{\mathrm{d}}$ National Centre for the Replacement, Refinement and Reduction of Animals in Research (NC3Rs), London, UK

${ }^{\text {e}}$ Covance Laboratories, Harrogate, UK

'Exponent International Limited, Harrogate, UK

${ }^{\mathrm{g}}$ The European Union Reference Laboratory for Alternatives to Animal Testing (EURL-ECVAM), European Commission Joint Research Centre, Ispra, Italy

${ }^{\mathrm{h}}$ Humane Society International, Research \& Toxicology Department, Brussels, Belgium

'European Partnership for Alternative Approaches to Animal Testing, Brussels, Belgium

\section{CITATION}

Moore, N. P., Andrew, D. J., Bjerke, D. L., Creton, S., Dreher, D., Holmes, T., ... \& Rowan, T. G. (2013). Can acute dermal systemic toxicity tests be replaced with oral tests? A comparison of route-specific systemic toxicity and hazard classifications under the Globally Harmonized System of Classification and Labelling of Chemicals (GHS). Regulatory Toxicology and Pharmacology,66(1), 30-37.

\section{KEYWORDS}

LD50, Reduction, Replacement, Acute systemic toxicity, Dermal, Oral, Rat, Classification

\section{ABSTRACT}

Acute systemic toxicity data (LD50 values) and hazard classifications derived in the rat following oral administration and dermal application have been analysed to examine whether or not orally-derived hazard classification or LD50 values can be used to determine dermal hazard classification. Comparing the oral and dermal classifications for 335 substances derived from oral and dermal LD50 values respectively revealed $17 \%$ concordance, and indicated that $7 \%$ of substances would be classified less severely while $76 \%$ would be classified more severely if oral classifications were applied directly to the dermal route. In contrast, applying the oral LD50 values within the dermal classification criteria to determine the dermal classification reduced the concordance to $15 \%$ and the relative 'under-classification' to $1 \%$, but increased the relative 'over-classification' to $84 \%$. Both under- and over-classification are undesirable, and mitigation strategies are discussed. Finally, no substance with an oral LD50 of >2000 $\mathrm{mg} / \mathrm{kg}$ was classified for acute systemic toxicity by the dermal route, suggesting that dermal testing for acute systemic toxicity of such substances adds nothing to the hazard characterisation and should be removed from routine regulatory data requirements.

${ }^{*}$ Corresponding author. Address: European Partnership for Alternative Approaches to Animal Testing, EPAA Industry Secretariat, Avenue Herrmann Debroux 15 B, B-1160 Brussels, Belgium. Fax: +32 2679 2019

E-mail address: tim_g_rowan@hotmail.com (T.G. Rowan).

${ }^{1}$ Current address: Environmental Protection Authority, Wellington, New Zealand. 


\section{Introduction}

The classification and labelling of chemicals is a mechanism for the identification and communication of intrinsic hazards of chemicals, enabling users to take appropriate protective measures. Such a system has been in place within the European Union since the entry into force of Council Directive 67/548/EEC, the 'Dangerous Substances Directive' (EEC, 1967). The United Nations later formulated a classification and labelling system for universal application, the Globally Harmonized System of Classification and Labelling of Chemicals (GHS). The GHS was first published in 2003 (UN, 2003), with the most recent revision published in 2011 (UN, 2011), and was enacted into the European legislative framework as Regulation 1272/2008 (EU, 2008).

The original European Directive established three categories of concern for acute systemic toxicity ('Very Toxic', 'Toxic', and 'Harmful'), whereas the GHS uses four or five numbered categories, depending on geographical jurisdiction. Both instruments established a categorisation procedure for determining the degree of concern attributed to the acute systemic toxicity of a substance based upon acute toxicity data. This is predominantly based on lethality values (e.g. LD50, LC50) established in laboratory experiments using animals. In both the European and GHS frameworks, the LD50 values that define the category boundaries differ between oral and dermal routes of exposure (Fig. 1).
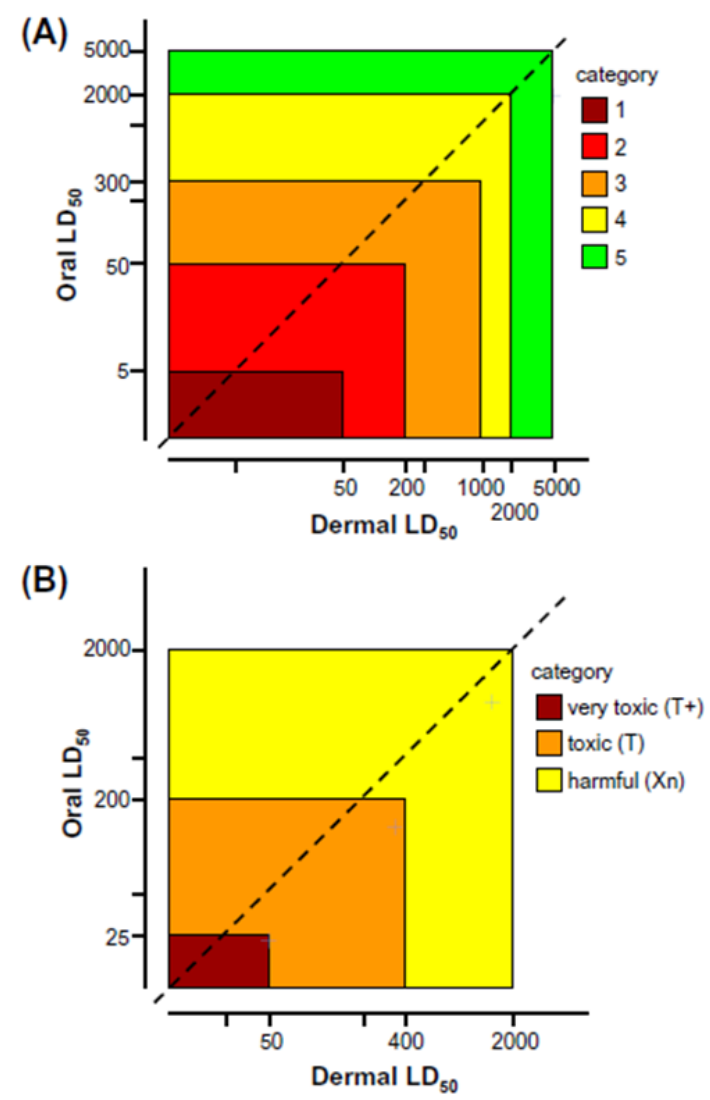

Fig. 1. The categories of classification for acute systemic toxicity by the oral and dermal routes of exposure. Classification categories are shown according to (A) GHS and (B) Council Directive 67/548/EEC (EEC, 1967). The dashed line represents the points at which the LD50 values for both routes are identical. LD50 values greater than 2000 or $5000 \mathrm{mg} / \mathrm{kg}$ (depending on regulatory framework) result in no classification under GHS; GHS category 5 is not obligatory and is not applied in all jurisdictions, e.g. within the European Union (EU, 2008). Axes are logarithmic.

It is not uncommon for substances to be tested by all three routes of exposure (oral, dermal, and inhalation), although studies may be waived if technically unfeasible or ethically undesirable, such as in the case of gases or corrosive substances. However, studies have shown that in the overwhelming 
majority of cases, classification by the dermal route is no more severe than by the oral route (Creton et al., 2010; Indans et al., 1998; Seidle et al., 2011; Thomas and Dewhurst, 2007); although one unpublished analysis has suggested a higher degree of 'false positives' (overclassification) and 'false negatives' (under-classification) by applying oral classifications to the dermal route, particularly when comparing oral classifications derived in the rat with dermal classifications derived in the rabbit (Paris et al., 2012).

Acute toxicity data are usually generated using standardized test methods, and the acute toxicity point estimate or range estimate is then used to determine the category of classification. The choice of species is likely to be restricted to those cited in the OECD test guidelines; the most commonly used species in the EU being the rat (OECD, 1987a, 2001a,b, 2008), although in some regions rabbits are more commonly used for dermal acute studies. Therefore, a comparison of acute toxicity data across species introduces an unnecessary complication, by adding interspecies differences in toxicokinetics and toxicodynamics to a comparison of systemic toxic potency consequent to route of administration. Given that one species, the rat, can be used to develop acute systemic toxicity data by both oral and dermal routes, it is most appropriate to restrict the comparison accordingly. The analysis presented here focuses on the experimental species most commonly used in Europe for both routes of exposure, the rat; considers the acute toxicity data itself, represented by the LD50 as point estimate; and compares the outcome to that obtained by comparing categories of classification. This work was conducted by the Acute Toxicity Working Group of the European Partnership for Alternative Approaches to Animal Testing (EPAA), as part of a wider evaluation of the necessity for acute toxicity testing and the development of alternative approaches without compromising safety.

\section{Data acquisition and analysis}

Acute systemic toxicity data for chemical substances administered to rats by the oral and dermal routes were acquired from the European Chemicals Agency's database (ECHA CHEM; accessed online at http://www.echemportal.org, on 24 May 2012) and similar data for pesticide actives were published by Creton et al. (2010). Data acquisition from ECHA CHEM was limited to experimental measurements of the LD50 in rats that were given a reliability rating of 1-"reliable without restriction"-or 2-"reliable with restrictions" (Klimisch et al., 1997).

As far as possible, data were normalised for gender, strain, and the concentration of active ingredient where applicable. All dose data were expressed in standard terms of milligrams of administered substance per kilogram bodyweight $(\mathrm{mg} / \mathrm{kg})$. Data were entered into Microsoft Excel for analysis.

Where the LD50 was quoted as a range of values, the lower limit of the range was taken. Acute toxicity studies derived to meet current classification requirements for substances of low intrinsic hazard may be conducted at a limit dose (generally $2000 \mathrm{mg} / \mathrm{kg}$ or $5000 \mathrm{mg} / \mathrm{kg}$ ). If less than $50 \%$ of the treated animals are killed, then the LD50 cannot be determined and it will be expressed as being greater than the limit dose used in the study. In such cases, the respective limit dose was used in place of the LD50, recognising that this represents an overestimation of toxicity of unknown proportion in most cases. Data were excluded from analysis where no meaningful comparison could be made, such as in the case of the LD50 by both routes exceeding limit values; point estimates by one route exceeding the limit dose used for the other route; or overlapping ranges of toxicity estimates.

Where the LD50 was quoted as being above the upper limit value for a specific category, the substance was classified into the next higher category. For example, a substance with LD50 > $1000-\mathrm{mg} / \mathrm{kg}$ was classified into category 4.

The category of classification for oral and dermal acute systemic toxicity for each substance was determined using the acute toxicity data acquired above, rather than relying on published classifications which may be based on other available data. These 'reference classifications' were derived using oral LD50 within the criteria for oral category definition and using dermal LD50 within the criteria for dermal category definition. The criteria for defining categories of classification are shown in Fig. 1A. The use of oral classification as a surrogate for dermal classification was evaluated by comparing the two reference 
classification categories directly. The use of oral toxicity data as a surrogate for dermal toxicity data was evaluated by applying the dermal classification criteria to the oral LD50 and comparing the derived classification category with the dermal reference classification.

The relative systemic toxic potency was estimated by dividing the oral LD50 by the dermal LD50.

\section{Results}

An initial dataset of 997 chemicals with oral and dermal LD50 values derived in the rat was developed, comprising 764 substances from ECHA CHEM and 233 pesticide actives (Creton et al., 2010). Classification according to the GHS five category system resulted in 279 substances classified into oral Category 5 and 822 substances classified into dermal Category 5. However, these classifications are largely the result of many substances not being tested at doses in excess of a limit value, and in many cases the upper limit dose was not the same for the two routes of administration. Dermal application was often limited to $2000 \mathrm{mg} / \mathrm{kg}$, while oral doses in excess of $5000 \mathrm{mg} / \mathrm{kg}$ were administered. No meaningful comparison of relative potency or classification can be made in such circumstances.

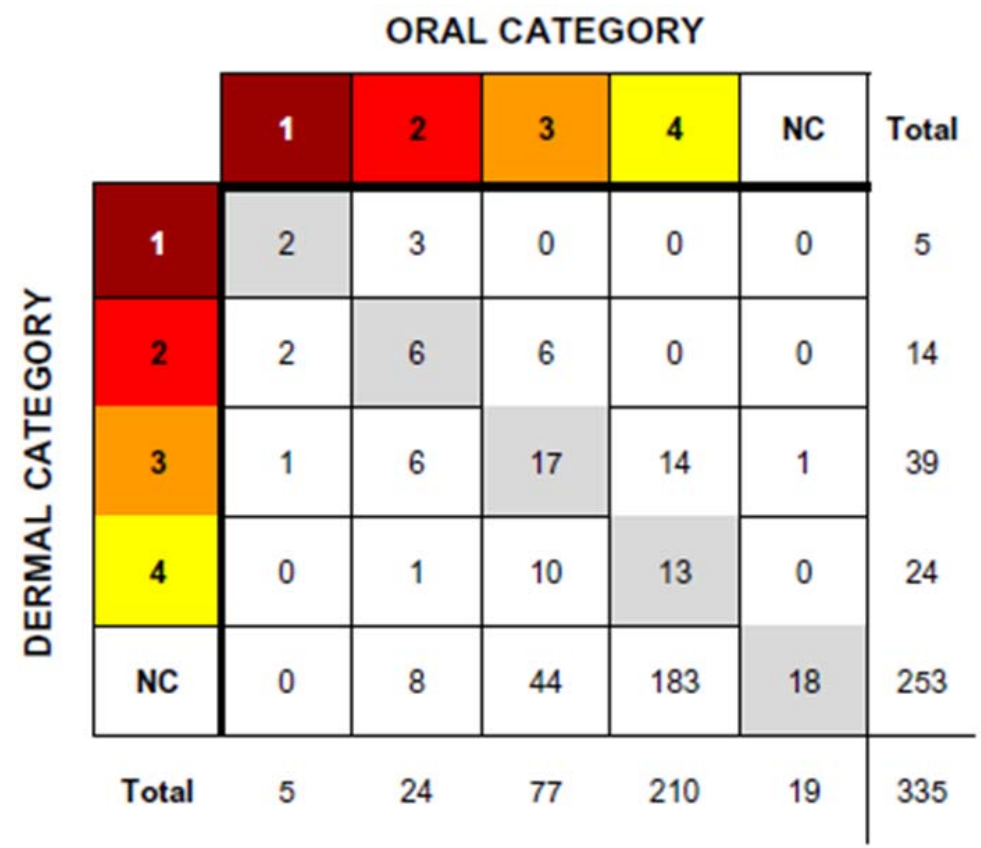

Table 1. Comparison of oral and dermal classifications. The oral and dermal classification categories under the four category GHS system (EU, 2008), that were assigned to 335 selected substances according to oral and dermal LD50 values derived in the rat. NC, not classified. The grey shading represents concordant outcomes.

Restricting the dataset to those substances for which relative potency by the oral and dermal routes could be estimated resulted in 335 substances, 225 from ECHA CHEM and 110 pesticide actives, the oral and dermal classification of which are given in Table 1. This dataset included eighteen substances for which LD50 point estimates were available but which were not classified for either route of administration, and omitted twenty-four classified substances for which the point estimate of the LD50 by one route exceeded the highest sub-LD50 dose tested by the other route, or where ranges of values overlapped (Table 2), so that a meaningful comparison could not be made; but overall, $95 \%$ of the substances were classified for acute toxicity by at least one route of administration (Table 1). In the majority of cases, the dermal LD50 exceeded the oral LD50 (Fig. 2A). For four substances, the relative systemic toxic potency ratio (i.e. oral LD50 divided by dermal LD50) was calculated to be 1, but this was largely the result of rounding limit data (Table 3), and in reality the ratios are lower. In five cases (2\%) the dermal LD50 was lower than the oral LD50 (Fig. 2B, Table 3). 
The majority of substances $(63 \%, n=210)$ were classified into Category 4 by the oral route, with $76 \%(n$ $=253$ ) not classified by the dermal route (Table 1). The proportion of substances that would be assigned a less severe category of classification ('underclassified') if the oral classification were also used as the dermal classification was $7 \%(n=24)$, the concordance was $17 \%(n=56)$, and in $76 \%$ of cases $(n=255)$ the oral classification would result in a more severe category of classification ('over-classified').

\begin{tabular}{|c|c|c|c|c|c|}
\hline \multirow[t]{2}{*}{ Substance } & \multicolumn{2}{|l|}{$\underline{L D_{50}}$} & \multicolumn{2}{|c|}{ Classification category } & \multirow[t]{2}{*}{ GHS local skin effects } \\
\hline & Dermal & Oral & Dermal & Oral & \\
\hline ECHA035 & $>200-<2000^{\mathrm{b}}$ & $200-500$ & 3 & 3 & Skin 2 \\
\hline ECHA058 & $>1080^{c}$ & 3227 & 4 & NC & \\
\hline ECHA069 & $>1157$ & 1784 & 4 & 4 & \\
\hline ECHA071 & $>75 \sigma$ & 2144 & 3 & NC & \\
\hline ECHA076 & $>1043-<4127$ & $>834-<1314$ & 4 & 4 & \\
\hline ECHA106 & $>1800-<3600$ & $>1400-<5000$ & 4 & 4 & Skin 2 \\
\hline ECHA109 & $\geqslant 934^{\mathrm{b}}$ & 2489 & 3 & NC & Skin 2 \\
\hline ECHA137 & $\geqslant 305$ & $\geqslant 90.4$ & 3 & 3 & Skin 1B \\
\hline ECHA156 & $>126.3^{c}$ & $>5000$ & 2 & NC & \\
\hline ECHA201 & $>1526^{c}$ & 2213 & 4 & NC & \\
\hline ECHA209 & $>400^{3<}$ & 630 & 3 & 4 & Skin 1C \\
\hline ECHA232 & $>1875$ & 3002 & 4 & NC & \\
\hline ECHA262 & $>1600^{c}$ & $>8300$ & 4 & NC & \\
\hline ECHA347 & $\geqslant 2000$ & $>1848$ & NC & 4 & \\
\hline ECHA531 & $>1000-<2000^{\circ}$ & 1591.4 & 4 & 4 & Skin 1B \\
\hline ECHA552 & $>132 \sigma^{\circ}$ & $>16000$ & 4 & NC & Skin 2 \\
\hline ECHA572 & $>1000-<2000^{\circ}$ & 1270 & 4 & 4 & Skin 1B \\
\hline PESTO4O & $43->2000$ & $24-85$ & 1 & 2 & \\
\hline PEST089 & $>2000$ & $1430-2490$ & NC & 4 & \\
\hline PEST122 & $>2000$ & $1800-2700$ & NC & 4 & \\
\hline PEST156 & 890 & $330-1720$ & 3 & 4 & \\
\hline PEST163 & $>2000$ & $>300(?)$ & NC & 4 & \\
\hline PEST206 & $>1640^{3}$ & $>4000$ & 4 & NC & "causes burns" \\
\hline PEST216 & 5000 & $>300$ & NC & 4 & \\
\hline
\end{tabular}

2 Skin 1A, 1B, 1C, corrosive; Skin 2, irritating. Not available for PEST (Creton et al., 2010).

Evidence of skin damage noted in study record (e.g. necrosis, oedema, erythema, inflammation).

c $L D_{0}$.

Table 2. Classified substances rejected from the assessment. Acute systemic toxicity data for 24 substances for which a quantitative comparison of systemic toxic potency by the oral and dermal routes could not be determined, and the classification categories assigned to them. Acute toxicity data were taken from ECHA CHEM or Creton et al. (2010). Classifications are according to the four category GHS system (EU, 2008). NC, not classified.

When the oral LD50 was applied within the dermal classification criteria to determine the dermal category of classification, the proportion of 'under-classified' substances was reduced to $1 \%(n=4)$ and concordance was reduced to $15 \%(n=50)$, while the proportion of substances that would be 'overclassified' increased to $84 \%(n=281)$. The number of substances originally identified as not classified was unchanged, but for classified substances there was a general shift to more severe acute dermal toxicity classifications in categories $1-4$, with most substances classified into category 3 (Table 4).

Twenty-eight substances appeared to be 'under-classified' for dermal hazard (Table 3). OneECHA117-was classified by the dermal route but not by the oral route irrespective of methodology. A further three-ECHA624, ECHA707, and PEST153-were 'underclassified' when the oral LD50 was used as a surrogate for the dermal LD50. All of these substances are classified as corrosive to skin, and the ECHA CHEM records for two of them reported necrosis at the site of application. For the remaining substances that appeared to be 'under-classified' when the oral classification was used directly as a surrogate for the dermal classification, this was a result of the disparity in category limits applied to hazard identification by the two routes; and for all except one of these cases-ECHA272, which is corrosive to skin-the dermal LD50 was greater than the oral LD50. Technical limitations in three cases-ECHA468, ECHA653, ECHA675-meant that the maximum dose that could be applied to the skin was well below the LD50 (specifically LD0 or LD10).

Of the twenty-four classified substances that were omitted from the semi-quantitative assessment of relative potency, nineteen were classified for dermal hazard and fifteen were classified for oral hazard. In many cases, the dermal classification was based on data that were limited in terms of the highest doses used (Table 2). 


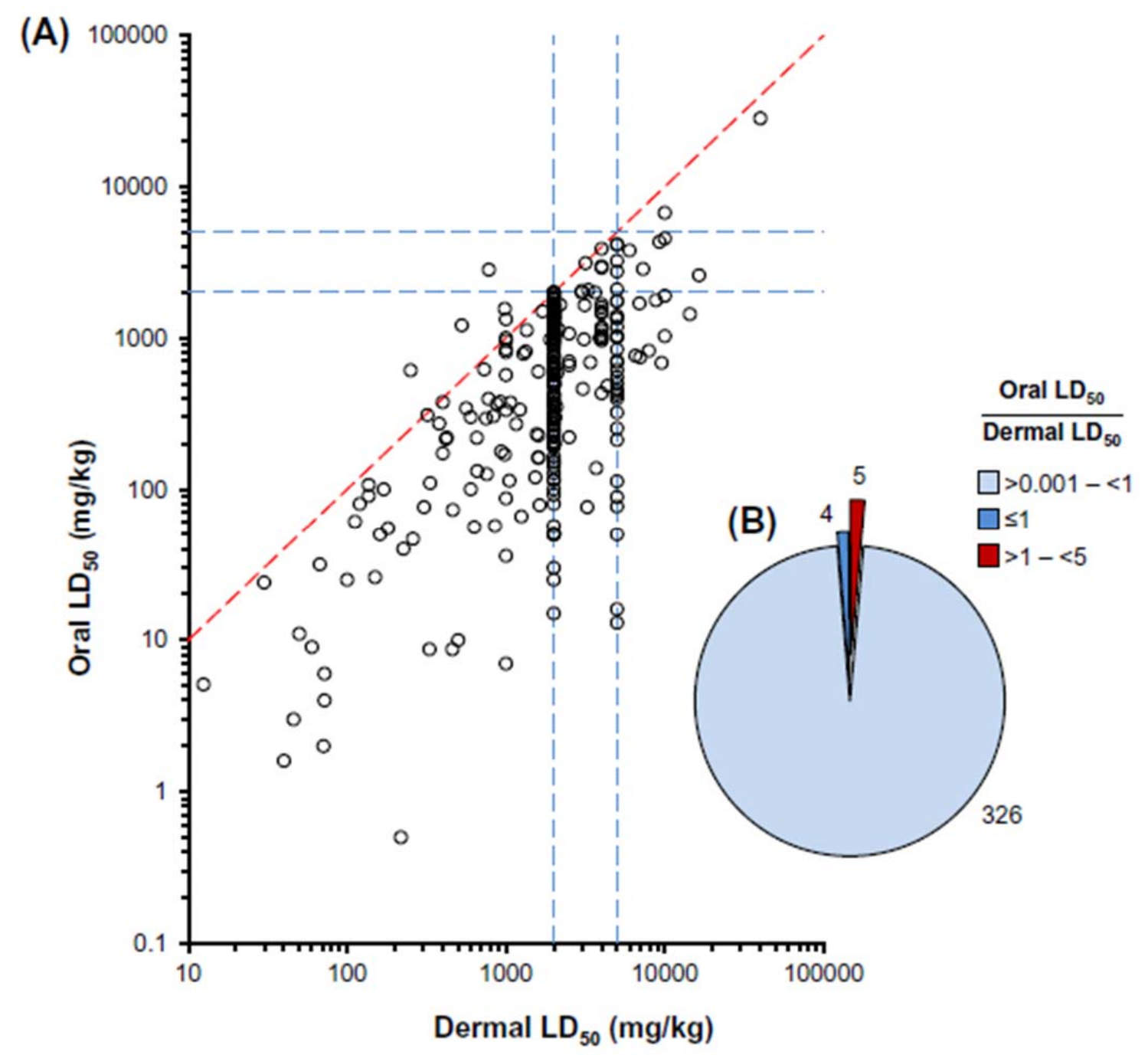

Fig. 2. Toxicant potency following oral and dermal administration of substances to the rat. (A) A scatter plot of concordance between estimates of oral and dermal systemic toxicity $(n=335)$. The diagonal dashed line represents the case where the LD50 values for the two routes are identical. The horizontal and vertical dashed lines represent the $2000 \mathrm{mg} / \mathrm{kg}$ and $5000 \mathrm{mg} / \mathrm{kg}$ dose levels that are often used as the upper limit of administration, and also represent the cut-off values for classification into categories 4 and 5 respectively; data points lying on these axes largely represent the maximum dose tested, and not the true estimate of the LD50 for that route of administration. (B) The relative systemic toxic potency following oral and dermal administration was estimated by dividing the oral LD50 by the dermal LD50. In the case of four substances for which this ratio is 1, it is overestimated (Table 3 ).

\section{Discussion}

The added value of acute dermal toxicity data in establishing the overall hazard profile of a substance has long been debated. In a review of 438 base set notifications, 90 substances were classified for acute oral toxicity, but only 4 were classified for acute dermal toxicity; and in only one instance (0.2\%) was classification for the dermal route more severe than for the oral route (Indans et al., 1998). This analysis was later updated to 1569 new substance registrations, of which 109 were classified for acute oral toxicity and 11 for acute dermal toxicity, with only one (0.06\%) substance having a more severe dermal classification than oral classification (Seidle et al., 2011). Similarly, of 195 pesticide actives evaluated by Thomas and Dewhurst (2007), only two (1\%) were of more severe classification by the dermal route than the oral route. These classifications were established according to the original criteria of Council Directive 67/548/EEC (EEC, 1967), rather than those of GHS (UN, 2003, 2011; EU, 2008). Of 337 pesticide actives classified under the GHS (without category 5), 182 were classified for oral toxicity and 51 for dermal 
toxicity. In $6(<2 \%)$ cases the dermal classification was more severe than the oral one (Seidle et al., 2011). However, the general conclusion by some authors that dermal classification does not influence the overall classification and labelling of substances in the majority of cases does not take into account the influence of dermal classification upon the implementation of exposure mitigation or risk management strategies, such as the use of personal protective equipment and the application of a 'skin notation' to a substance with an occupational exposure limit (Chen et al., 2011; Lavoué et al., 2008).

\begin{tabular}{|c|c|c|c|c|c|c|c|}
\hline \multirow[t]{2}{*}{ Substance } & \multicolumn{3}{|l|}{$L_{50}$} & \multicolumn{3}{|c|}{ Classification category } & \multirow[t]{2}{*}{ GHS local skin effects } \\
\hline & Dermal & Oral & Ratio & Dermal & Oral & Dermal (oral LDso) & \\
\hline ECHA116 & 921 & 379 & 0.41 & 3 & $4^{\circ}$ & 3 & \\
\hline ECHA117 & $780^{\mathrm{b}}$ & 2830 & 3.63 & 3 & $\mathrm{NC}^{*}$ & $\mathrm{NC}^{*}$ & Skin 1B \\
\hline ECHA120 & ca. 1000 & 806 & 0.81 & 3 & $4^{\circ}$ & 3 & Skin 1B \\
\hline ECHA157 & $777^{\circ}$ & 396 & 0.51 & 3 & $4^{*}$ & 3 & Skin 2 \\
\hline ECHA159 & $>170-<200$ & 100 & 0.59 & 2 & $3^{\circ}$ & 2 & Skin 2 \\
\hline ECHA171 & 30 & 24 & 0.80 & 1 & $2^{*}$ & 1 & Skin 2 \\
\hline ECHA228 & 320 & 310 & 0.97 & 3 & $4^{\circ}$ & 3 & \\
\hline ECHA272 & $251^{\mathrm{D}}$ & 612 & 2.44 & 3 & $4^{\circ}$ & 3 & Skin 1B \\
\hline ECHA410 & ca. $730^{\circ}$ & ca. 620 & 0.85 & 3 & $4^{\circ}$ & 3 & Skin 2 \\
\hline ECHA414 & $>400-<2000^{3}$ & 377.1 & 0.94 & 3 & $4^{\circ}$ & 3 & Skin 1B \\
\hline ECHA468 & $>1000^{\mathrm{b} C \mathrm{c}}$ & ca. 1001 & $\leqslant 1.00$ & 4 & 4 & 4 & Skin 1B \\
\hline ECHA486 & $136.6^{\mathrm{b}}$ & 90.4 & 0.66 & & $3^{\circ}$ & 2 & Skin 1B \\
\hline ECHA554 & ca. 12.45 & ca. 5.1 & 0.41 & 1 & $2^{*}$ & 1 & Skin 1B \\
\hline ECHA624 & 527 & 1215 & 2.31 & 3 & $4^{\circ}$ & $4^{\circ}$ & Skin 1B \\
\hline ECHA637 & ca. 1000 & 841 & 0.84 & 3 & $4^{\circ}$ & 3 & Skin 1B \\
\hline ECHA653 & $>875^{\circ}$ & 367.3 & 0.41 & 3 & $4^{\circ}$ & 3 & \\
\hline ECHA657 & $181^{\circ}$ & 55 & 0.30 & 2 & $3^{*}$ & 2 & Skin 1B \\
\hline ECHA664 & $136.6^{\mathrm{b}}$ & 107 & 0.78 & 2 & $3^{\circ}$ & 2 & Skin 2 \\
\hline ECHA675 & $>2000^{6}$ & 2000 & $\leqslant 1.00$ & $\mathrm{NC}$ & 4 & 4 & \\
\hline ECHA678 & 833 & 304 & 0.36 & 3 & $4^{\circ}$ & 3 & \\
\hline ECHA707 & $<1000^{\mathrm{b}}$ & 1330 & 1.33 & 3 & $4^{\circ}$ & $4^{\circ}$ & Skin 1C \\
\hline PEST015 & $>2000$ & $<2000$ & $\leqslant 1.00$ & NC & 4 & 4 & \\
\hline PESTO49 & $562-800$ & $343-556$ & 0.61 & 3 & $4^{\circ}$ & 3 & \\
\hline PEST083 & 50 & 11 & 0.22 & 1 & $2^{*}$ & 1 & \\
\hline PEST 147 & 120 & 80 & 0.66 & 2 & $3^{\circ}$ & 2 & \\
\hline PEST153 & 983-1390 & $1560-2210$ & 1.59 & 3 & $4^{\circ}$ & $4^{\circ}$ & "causes burns" \\
\hline PEST169 & 112 & 61 & 0.54 & 2 & $3^{\circ}$ & 2 & \\
\hline PEST174 & $>2000$ & 2000 & $\leqslant 1.00$ & NC & 4 & 4 & \\
\hline
\end{tabular}

Table 3. Substances apparently 'under-classified' by applying the oral classification or data to the dermal route. Acute systemic toxicity data and hazard classifications for 28 substances that would be under-classified for dermal hazard based on oral data or classification. Also included are four borderline cases for which the acute systemic toxic potency ratio is estimated to be less than or equal to unity. Acute toxicity data were taken from ECHA CHEM or Creton et al. (2010). Classifications are according to the four category GHS system (EU, 2008). NC, not classified.

\subsection{Challenges associated with comparing route-specific classifications}

The direct comparison of classifications by route is not without some complications.

The cut-off doses for classification within given categories differ for the oral and dermal routes (Fig. 1). For a given dose or exposure level, classification for the dermal route is more severe than for the oral route in many cases. Under the GHS, if the oral and dermal LD50 values are $5 \mathrm{mg} / \mathrm{kg}$ or less, in the range 200-300 mg/kg, or greater than $1000 \mathrm{mg} / \mathrm{kg}$, the classification category is the same for both routes of administration. However, when the LD50 is in the range $5-50 \mathrm{mg} / \mathrm{kg}$, classification by the oral route falls into category 2 whereas by the dermal route it is category 1 ; in the range $50-200 \mathrm{mg} / \mathrm{kg}$ the oral and dermal classifications are for category 3 and 2 respectively; and in the range 300-1000 mg/kg the oral and dermal classifications are for category 4 and 3 respectively. For example, consider the case for substance ECHA678 (Table 3), with an oral LD50 value of $304 \mathrm{mg} / \mathrm{kg}$ and a dermal LD50 of $833 \mathrm{mg} / \mathrm{kg}$, which is 2.7-fold higher; under GHS this substance would be classified into category 4 for oral toxicity, yet into category 3 for toxicity via the dermal route. In fact, many of the apparent 'misclassifications' observed in this analysis are the result of the disparity in category limits between oral and dermal hazard classification schemes (Table 3). 
Additional shortcomings associated with comparing published classifications (Indans et al., 1998; Seidle et al., 2011; Thomas and Dewhurst, 2007), as opposed to those derived from a specified dataset (Creton et al., 2010; Paris et al., 2012; Seidle et al., 2011), relate to a number of other confounding factors that are unlikely to be relevant in the testing of a new substance.

Firstly, during the classification process, it is common to use the most conservative (i.e. lowest) value of the LD50-unless there is valid reason to do otherwise. This means that published classifications may be based on oral and dermal acute toxicity data derived from different species, including human experience, or investigative studies. Comparing hazard classifications for different routes of exposure using data from different species further complicates the analysis, by introducing an additional response variable. Secondly, reports of fatality or non-fatal morbidity in acute human poisoning cases generally focus on single individuals presenting to emergency services or medical professionals; in such cases, exposure to the substance may be estimated to a greater or lesser degree of accuracy. When such information is used as the basis for classification, the LDLO-and in some cases EDLO-will be used in place of LD50 data. $^{2}$ Whereas the LD50 offers an estimate of the median response of a population, the LDLO and EDLO only reflect reports on the most susceptible individuals that have been exposed to a substance. Thirdly, where GHS classifications are derived from earlier classifications, for example, using a 'translation table', misclassifications may arise as a result of the different cut-off doses used to define classification categories in different classification schemes (Fig. 1). These published classifications can only be amended on a case-by-case basis, by reference to the underlying toxicity data. ${ }^{3}$ Finally, some degree of imprecision exists in classification based on acute toxicity estimates (Hoffmann et al., 2010; OECD, 2001a,b), as discussed further below.

The attraction of comparing classification categories rather than LD50 values is that it readily accommodates those cases where acute toxicity was estimated on the basis of a 'class method'. Furthermore, classifications for substances are more readily available than the underlying toxicity information, which in many cases is not in the public domain or in a readily amenable format.

\subsection{Classification is inherently inexact}

It is apparent that the disparity between categories of classification for the oral and dermal routes is in part due to the different criteria (LD50 cut-off values) used to establish the categories, which can result in more severe classifications for the dermal route. This confounder is highlighted when the dermal classification criteria are applied to oral LD50 values, with the outcome that classification is increased in severity. This observation belies the generally greater toxic potency of substances when administered by the oral route compared to the dermal route (Fig. 2).

It should be noted that the direct comparison of oral and dermal LD50 data only gives an approximation of the relative potency, since the values are often not derived under the same conditions. Even point estimates have a degree of variability in their estimation (Hoffmann et al., 2010; Lipnick et al., 1995; Weil, 1975); statistical modelling of oral LD50 variability indicated that while most substances would fall into only one GHS category, a substantial proportion (44\%) would fall within two adjacent categories (Hoffmann et al., 2010). Furthermore, in many cases the estimate of the dermal LD50 was defined by a limit dose (Fig. 2A). Consequently, while care has been taken in the current analysis to ensure that the relative potency by route is unchanged in relation to parity-for example, substances with overlapping ranges for oral and dermal LD50 values were omitted, without prejudice to the assessment as discussed later-the magnitude of the ratios may be subject to variability in some cases and therefore they are not presented (Fig. 2B).

\footnotetext{
${ }^{2}$ LDLO and EDLO refer to the lowest (estimated) dose or level of exposure resulting in either lethality or non-lethal toxicity of serious concern, respectively.

${ }^{3}$ Regulation (EC) No 1272/2008, Annex VI, 1.2.1 (EU, 2008).
} 
Current test guidelines for the determination of acute oral toxicity-particularly OECD TG 420 and 423 (OECD 2001a,b) - are designed to determine the category of classification for a substance rather than to establish the LD50 per se; although the Up-and-Down Procedure, OECD TG 425, does allow the LD50 to be estimated (Lipnick et al., 1995; OECD, 2008). As such, the dose associated with the causation of acute toxicity might only be established to the extent needed to determine the category of classification, which may itself be influenced by the response in a single animal. As noted earlier, the cut-off doses for determining the severity of hazard classification differ for the oral and dermal routes (Fig. 1). This means that some ambiguity exists in translating oral classifications to the dermal route if a 1:1 toxicity relationship is assumed. However, lethality can be taken as an indicator of systemic exposure following treatment by any route of administration-toxicokinetic differences other than absorption notwithstanding - and it is therefore apparent that systemic loading following dermal application is predominantly lower than that following oral administration, although this may in part reflect methodological limitations of the tests themselves (e.g. excessive skin surface loading at high doses resulting in lower systemic availability of the applied dose).

\subsection{Approaches to extrapolating oral hazard to the dermal route}

If the requirement for dermal acute systemic hazard classification is to be served by the use of oral toxicity data, then there are two feasible procedures by which this might be accomplished. The first is to simply use the oral classification category as a surrogate for the dermal classification category. The second is to use the oral LD50 as a surrogate for the dermal LD50 and then apply the dermal classification criteria using this surrogate value. Both of these approaches have been evaluated in the present communication, using the same dataset.

Direct application of the oral category to the dermal classification resulted in $7 \%(n=24)$ of substances being 'under-classified', and $76 \%$ being 'over-classified' (Table 1); using the oral LD50 to derive the dermal classification resulted in $1 \%(n=4)$ of substances being 'under-classified', whilst $84 \%$ were 'overclassified' (Table 4). However, it must be recognised that classification for acute systemic toxicity is an imprecise procedure, and that a certain degree of inconsistency is inherent. For example, classification for oral toxicity using the Fixed Dose Procedure and Acute Toxic Class methods may result in the category of classification being determined by the response in a single animal (OECD, 2001a,b). Furthermore, as noted earlier, Hoffmann et al. (2010) have shown that variability in the determination of oral LD50 values indicates that $54 \%$ of substances would fall into one category of classification for oral systemic acute toxicity while $44 \%$ would fall into one of two adjacent categories. If these considerations are taken as an indicator of the expected reliability of oral classification-that is within two adjacent categories in the majority of cases-then the degree of concordance between oral and dermal classification approaches $84 \%$, and only one substance (0.3\%) remains 'under-classified' (Table 1).

Twenty-eight substances were apparently 'under-classified' for dermal hazard (Table 3). Many of these substances are classified as corrosive or irritating to the skin and the ECHA CHEM records in some cases reported necrosis or non-necrotic changes at the site of application. Even non-necrotic changes to the skin (e.g. erythema, oedema, inflammation, fissuring) can compromise the skin barrier and increase the trans-dermal transfer of xenobiotics. Such conditions should not arise in modern dermal toxicity studies conducted to acceptable standards of animal welfare. Other reasons why substances may be 'under-classified' by the dermal route are the disparity in category limits applied to hazard identification by the two routes (discussed earlier) and technical limitations in test substance application to the skin. Substances for which the maximum possible applicable dose was below the LD50 would not be classified for acute systemic toxicity by the dermal route based on weight-of-evidence. Consequently, substances that were apparently 'under-classified' for dermal hazard were either damaging to the integrity of the skin barrier, reflected differences in category definition, or would not be classified on a weight-of-evidence basis. None of these examples are judged to represent unique systemic toxicity hazards presented by application to the skin.

Twenty-four classified substances were omitted from the analysis, because the acute systemic toxic potency following exposure by the oral and dermal routes could not be meaningfully compared (Table 2). Of these, 12 were assigned a more severe category of classification for dermal hazard than for oral 
hazard, three of which-ECHA109, ECHA209, and PEST206-are extremely irritating or corrosive to the skin and caused damage to the site of application. For an additional seven substances-ECHA058, ECHA071, ECHA156, ECHA201, ECHA232, ECHA262, and ECHA552, as well as ECHA209-the quoted lower bound for the LD50 is in reality the LD0; overall, on the basis of weight of evidence, these substances would not be classified for systemic toxicity by the dermal route. There were no deaths among rats administered $1080 \mathrm{mg} / \mathrm{kg}$ ECHA058, a substance for which the dermal lethal dose in rabbits is reported to exceed $2000 \mathrm{mg} / \mathrm{kg}$. ECHA201 was tested as a $65 \%$ solution applied to the skin at a dose level of $2000 \mathrm{mg} / \mathrm{kg}$ in one study and $2348 \mathrm{mg} / \mathrm{kg}$ in a second study; there were no mortalities, and when expressed in terms of the material as tested, rather than the active, the product is not classified. Similarly, ECHA071 was classified into category 3 on the basis of LD50 $>750 \mathrm{mg} / \mathrm{kg}$, although there were no deaths at this dose level; the LD50 in rabbit is quoted as $>20000 \mathrm{mg} / \mathrm{kg}$, while a rat study with a reliability rating of 4-"reliability not assignable" (Klimisch et al., 1997)—quotes LD50 > $16000 \mathrm{mg} / \mathrm{kg}$. The one substance classified into category 2 for dermal toxicity, ECHA156, is a UV absorbant used in sunscreens, and was classified on the basis of a very low application in a sunscreen cream formulation; there were no deaths in 5 male and 5 female rats administered $5 \mathrm{~mL} / \mathrm{kg}$ of a preparation containing $<10 \%$ of the active ingredient (LD50 > $126 \mathrm{mg} / \mathrm{kg}$ ). ECHA232 caused no mortalities at a dose of $2 \mathrm{~mL} / \mathrm{kg}$, the highest dose that could be applied to the skin; the LD50 in rabbit is $>13000 \mathrm{mg} / \mathrm{kg}$. ECHA262 was tested in two studies, in which $1600 \mathrm{mg} / \mathrm{kg}$ and $2000 \mathrm{mg} / \mathrm{kg}$ were applied to the skin without mortality. Finally, ECHA552 was applied at the highest dose technically feasible (25\% in vehicle) without mortality. Two other substances in this group-PEST040 and PEST156-were of greater toxic potency by the oral route, but once again were classified more stringently by the dermal route. Consequently, of the twelve substances that might be under-classified for dermal hazard using the criteria outlined earlier, three might be expected to cause some degree of skin damage, two were assigned different hazard categories on the basis of differences in category definition, and the remainder would not be classified for dermal hazard on the basis of weight-of-evidence. Again, none of these examples are judged to represent unique systemic toxicity hazards presented by application to the skin, and their exclusion is not deemed to have had a negative impact on the analysis.

\subsection{Implications of using oral data to assign the dermal hazard classification}

It is clear that the dermal hazard classification does not drive the overall classification for the substances evaluated herein, a conclusion that is in agreement with previous analyses (Creton et al., 2010; Indans et al., 1998; Seidle et al., 2011; Thomas and Dewhurst, 2007). But while under-classification is obviously a concern, resulting in a lower degree of protection, over-classification is also undesirable, since it may lead to users' desensitisation to real hazards, product deselection, or the application of unwarranted exposure controls. The two methods presented herein for using oral data to determine dermal hazard category result in both under- and over-classification, although imprecision is inherent to the classification process as discussed above. Consequently, it is likely that neither of these approaches could suffice as they stand in all circumstances, and consideration should be given to how they may be adapted or incorporated into a testing strategy.

Both approaches result in a high degree of false positives ('overclassification'), but the use of the oral LD50 as a surrogate for the dermal LD50 resulted in a lower degree of false negatives ('underclassification'), previously described considerations on the precision of categorisation notwithstanding. Using the oral classification as a direct surrogate for the dermal classification introduces further complications, due to the differences in the boundaries that define the categories for the two routes, in addition to route-related differences in toxic potency. So taking the oral LD50 and applying dermal classification criteria seems to be a reasonable starting position for determining dermal hazard. Since the deletion of the original OECD test guideline for acute oral toxicity, OECD test guideline 401 (OECD, 1987b), a point estimate for the LD50 of substances is only available if oral toxicity is determined with the Up-and-Down Procedure (OECD, 2008); otherwise, the use of other class methods would require an approximation to be used, such as the lower bound of a category (OECD, 2001a,b) or 'LD50 cutoff' (OECD, 2001b). Since the greater toxic potency of xenobiotics administered by the oral route lies, at least in part, in the barrier effect of the skin reducing systemic exposure as compared to the oral route, the oral LD50 (point value or lower bound estimate) might be modified by information on dermal penetration (Marzulli et al., 1969; Mostert and Goergens, 2011), either experimentally derived in vitro or determined 
by a suitably validated model or read-across from structural analogues. Current models for dermal penetration, however, have been developed and validated using data derived from studies with human skin samples (Guy, 2010; Mitragotri et al., 2011; Potts and Guy, 1992; ten Berge, 2009), while there is some evidence to suggest that rat epidermis is more permeable than that of humans under like conditions (ECETOC, 1993; Garnett et al., 1994; Kenyon et al., 2004; van Ravenzwaay and Leibold, 2004a,b). Consequently, the available models would underestimate dermal penetration-and therefore systemic toxicity-in the rat, and a species-specific model is needed before an in silico approach could be adopted. Although in vitro dermal penetration tests are commonly more expensive to conduct than in vivo acute systemic toxicity tests, they have greater utility in risk assessment and study design, while the acute toxicity test is more limited in its applicability. Should the need for in vivo data be unavoidable, this strategy might prove useful in limiting the extent of work needed. Furthermore, the current OECD test guideline for acute dermal systemic toxicity testing (OECD, 1987a) could be revised, similar to the guidelines for acute oral systemic toxicity testing (OECD, 2001a,b, 2008), 4 to reduce the use of animals should testing be required (Creton et al., 2010; Diener et al., 1998; Stallard et al., 2004).

\section{DERMAL CATEGORY BASED ON ORAL LD 50}

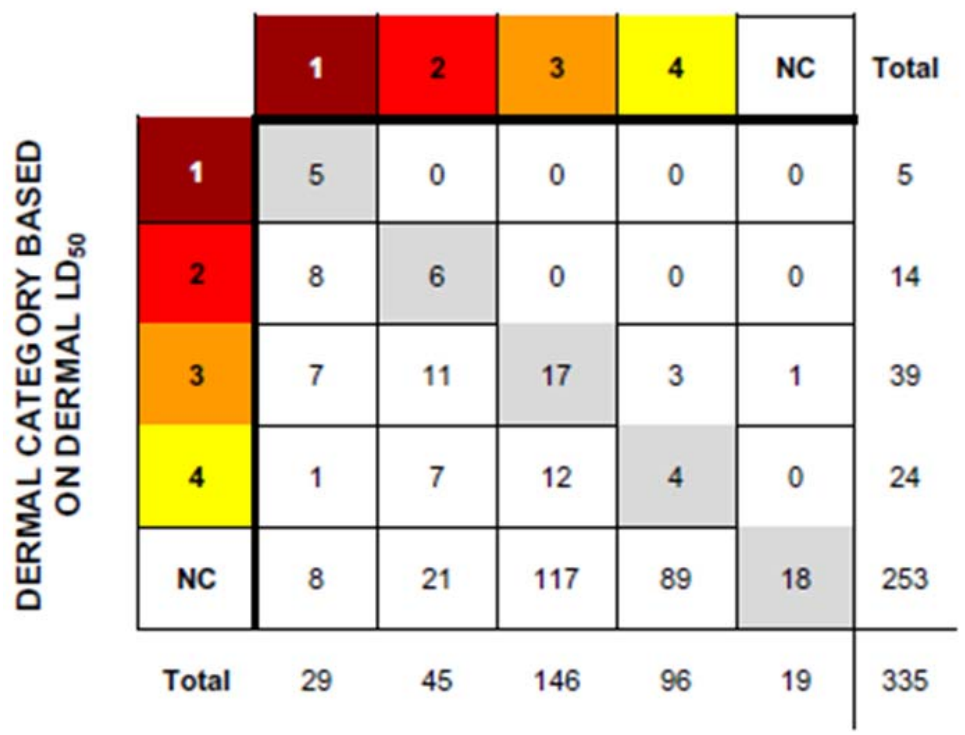

Table 4. Comparison of dermal classifications based on dermal and oral toxicity data. The categories of classification for acute systemic toxicity by the dermal route that were assigned to the 335 selected substances in Table 3 , based on dermal and oral LD50 values derived in the rat and using the category criteria for dermal classification under the four category GHS system (EU, 2008). NC, not classified. The grey shading represents concordant outcomes.

Of the 997 substances originally selected from the ECHA CHEM database and Creton et al. (2010), 672 had an oral LD50 $>2000 \mathrm{mg} / \mathrm{kg}$, of which only 10 had a dermal LD50 $<2000 \mathrm{mg} / \mathrm{kg}$. These substances are shown in Table 2 (ECHA058, ECHA071, ECHA109, ECHA156, ECHA201, ECHA232, ECHA262, ECHA552, and PEST206) and Table 3 (ECHA117), and have been evaluated above. All of these substances are either strongly irritating or corrosive to the skin, or would not be classified for dermal hazard based on weight of evidence. Consequently, testing a substance with an oral LD50 value of $>2000$ $\mathrm{mg} / \mathrm{kg}$ by the dermal route will not add to its hazard characterisation (under the four category GHS system), and should be discontinued in Europe in accordance with the requirements for the protection of animals used for scientific purposes (EU, 2010).

${ }^{4} \mathrm{As}$ of this time of writing, an Up-and-Down Procedure for acute dermal systemic toxicity testing is being evaluated by NICEATM/ICCVAM (Anon, 2012). 


\section{Conclusion}

Direct comparison of oral and dermal acute systemic toxicity for a broad spectrum of chemicals tested has been conducted. Such analyses may be confounded to a certain extent by factors such as interspecies differences in susceptibility; methodological difficulties in testing (e.g. dose-limiting physical chemistry under dermal application); different batches of chemical being tested; dose-limiting local toxicity under dermal application (i.e. corrosion, irritation); and differences in the bounding cut-off LD50 values defining categories for oral and dermal classification. Such confounders have been limited to the extent possible in this analysis. Overall, we conclude that toxicity of chemicals is greater by the oral route than the dermal route and that oral acute systemic toxicity data can be used in place of equivalent dermal testing, with little or no concern for under-classification, by application of the dermal classification criteria. This approach does increase the potential for over-classification, which may be ameliorated by consideration of dermal penetration. Furthermore, testing a substance with an oral LD50 value of $>2000$ $\mathrm{mg} / \mathrm{kg}$ by the dermal route will not add to its hazard characterisation and should be dissuaded.

\section{Conflict of interest}

Other than stated employment, the authors are not aware of any conflicts of interest. This work was conducted under the auspices of the European Partnership for Alternative Approaches to Animal Testing (EPAA, http://ec.europa.eu/enterprise/epaa/index_en.htm), a voluntary collaboration between the European Commission, European trade associations, and companies from seven industry sectors.

\section{Acknowledgments}

The authors wish to thank Dr Gabriele Küsters (PIC) for project management, and Drs René Hunziker (Dow Europe) and Dan Wilson (Dow Chemical Company) for technical review.

\section{References}

Anon., 2012. Evaluation of an Up-and-Down procedure for acute dermal systemic toxicity testing: request for nominations for an independent expert panel and submission of relevant data. Fed. Reg. 77, 43089 [Online]. Available at: <http://iccvam.niehs.nih.gov/methods/acutetox/udp-dermal.htm>. Accessed 26.11.2012.

Chen, C.P., Ahlers, H.W., Scott Dotson, G., Lin, Y.C., Chang, W.C., Maier, A., Gadagbui, B., 2011. Efficacy of predictive modeling as a scientific criterion in dermal hazard identification for assignment of skin notations. Regul. Toxicol. Pharmacol. 61, 63-72.

Creton, S., Dewhurst, I.C., Earl, L.K., Gehen, S.C., Guest, R.L., Hotchkiss, J.A., Indans, I., Woolhiser, M.R., Billington, R., 2010. Acute toxicity testing of chemicals-opportunities to avoid redundant testing and use alternative approaches. Crit. Rev. Toxicol. 40, 50-83.

Diener, W., Kayser, D., Schlede, E., 1998. The dermal acute toxic class method: test procedures and biometric evaluations. Arch. Toxicol. 72, 751-762.

ECETOC, 1993. Percutaneous Absorption. In: Monograph 20. The European Centre for Ecotoxicology and Toxicology of Chemicals, Brussels, ISSN-0773-6347-20.

EEC, 1967. Council Directive 67/548/EEC of 27 June 1967 on the approximation of laws, regulations and administrative provisions relating to the classification, packaging and labelling of dangerous substances. Off. J. Eur. Commun. 196, 1-98, 16.8.1967.

EU, 2008. Regulation (EC) No 1272/2008 of the european parliament and of the council of 16 december 2008 on classification, labelling and packaging of substances and mixtures, amending and repealing directives 67/548/eec and 1999/45/ec, and amending regulation (ec) No 1907/2006. Off. J. Eur. Commun. L 353, 1-1355, 31.12.2008.

EU, 2010. Directive 2010/63/EU of the European Parliament and of the Council of 22 September 2010 on the protection of animals used for scientific purposes. Off. J. Eur. Commun. L 276, 33-79, 20.10.2010.

Garnett, A., Hotchkiss, S.A.M., Caldwell, J., 1994. Percutaneous absorption of benzyl acetate through rat skin in vitro. 3. A comparison with human skin. Food Chem. Toxicol. 32, 1061-1065. 
Guy, R.H., 2010. Predicting the rate and extent of fragrance chemical absorption into and through the skin. Chem. Res. Toxicol. 23, 864-870.

Hoffmann, S., Kinsner-Ovaskainen, A., Prieto, P., Mangelsdorf, I., Bieler, C., Cole, T., 2010. Acute oral toxicity: variability, reliability, relevance and interspecies comparison of rodent LD50 data from literature surveyed for the ACuteTox project. Regul. Toxicol. Pharmacol. 58, 395-407.

Indans, I., Fry, T., Evans, P., 1998. Classification and labelling of new industrial chemicals for acute toxicity, skin and eye irritation. Hum. Exp. Toxicol. 17, 529.

Kenyon, S., Carmichael, P.L., Khalaque, S., Panchal, S., Waring, R., Harris, R., Smith, R.L., Mitchell, S.C., 2004. The passage of trimethylamine across rat and human skin. Food Chem. Toxicol. 42, 1619-1628.

Klimisch, H.J., Andreae, M., Tillmann, U., 1997. A systematic approach for evaluating the quality of experimental toxicological and ecotoxicological data. Regul. Toxicol. Pharmacol. 25, 1-5.

Lavoué, J., Milon, A., Droz, P.O., 2008. Comparison of indices proposed as criteria for assigning skin notation. Ann. Occup. Hyg. 52, 747-756.

Lipnick, R.L., Cotruvo, J.A., Hill, R.N., Bruce, R.D., Stitzel, K.A., Walker, A.P., Chu, I., Goddard, M., Segal, L., Springer, J.A., Myers, R.C., 1995. Comparison of the up-and-down, conventional LD50, and fixed dose acute toxicity procedures. Food Chem. Toxicol. 33, 223-231.

Marzulli, F.N., Brown, D.W.C., Maibach, H.I., 1969. Techniques for studying skin penetration. Toxicol. Appl. Pharmacol. 14 (Suppl. 3), 76-83.

Mitragotri, S., Anissimov, Y.G., Bunge, A.L., Frasch, H.F., Guy, R.H., Hadgraft, J., Kasting, G.B., Lane, M.E., Roberts, M.S., 2011. Mathematical models of skin permeability: an overview. Int. J. Pharm. 418, 115-129.

Mostert, V., Goergens, A., 2011. Dermal DNEL setting: using QSAR predictions for dermal absorption for a refined route-to-route extrapolation. Toxicologist 120, 107 (abstract \#499).

OECD, 1987a. Acute Dermal Toxicity. OECD Guideline for the Testing of Chemicals, vol. 402. OECD, Paris.

OECD, 1987b. Acute Oral Toxicity. OECD Guideline for the Testing of Chemicals, vol. 401. OECD, Paris.

OECD, 2001a. Acute Oral Toxicity - Fixed Dose Procedure. OECD Guideline for the Testing of Chemicals, vol. 420. OECD, Paris.

OECD, 2001b. Acute Oral Toxicity - Acute Toxic Class Method. OECD Guideline for the Testing of Chemicals, vol. 423. OECD, Paris.

OECD, 2008. Acute Oral Toxicity - Up-and-Down-Procedure (UDP). OECD Guideline for the Testing of Chemicals, vol. 425. OECD, Paris.

Paris, M., Strickland, J., Stack, F., Allen, D., Casey, W., Stokes, W., 2012. Analysis to determine if acute oral systemic toxicity data can be used to estimate and avoid acute dermal systemic toxicity testing. Toxicologist 126, 399, abstract \#1856.

Potts, R.O., Guy, R.H., 1992. Predicting skin permeability. Pharm. Res. 9, 663-669.

Seidle, T., Prieto, P., Bulgheroni, A., 2011. Examining the regulatory value of multiroute mammalian acute systemic toxicity studies. ALTEX 28, 95-102.

Stallard, N., Whitehead, A., Indans, I., 2004. Statistical evaluation of an acute dermal toxicity test using the dermal fixed dose procedure. Hum. Exp. Toxicol. 23, 405-412.

ten Berge, W., 2009. A simple dermal absorption model: derivation and application. Chemosphere 75, 1440-1445.

Thomas, H.D., Dewhurst, I.C., 2007. What does a dermal acute toxicity study add to the information on a plant protection pesticide? Toxicology 231, 114-115.

UN, 2003. Globally Harmonized System of Classification and Labelling of Chemicals (GHS). UN Economic Commission for Europe, Geneva.

UN, 2011. Globally Harmonized System of Classification and Labelling of Chemicals (GHS). UN Economic Commission for Europe, Geneva, http://www.unece.org/trans/danger/publi/ghs/ghs_rev04/04files_e.html. Accessed 2.5.2012.

van Ravenzwaay, B., Leibold, E., 2004a. The significance of in vitro rat skin absorption studies to human risk assessment. Toxicol. In vitro. 18, 219-225.

van Ravenzwaay, B., Leibold, E., 2004b. A comparison between in vitro rat and human and in vivo rat skin absorption studies. Hum. Toxicol. 23, 421-430.

Weil, C.S., 1975. Toxicology experimental design and conduct as measured by interlaboratory collaborative studies. J. Assoc. Off. Anal. Chem. 58, 683-688. 